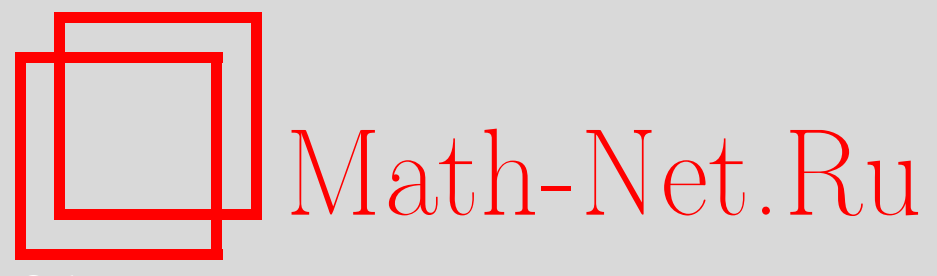

В. А. Емеличев, Р. А. Бердышева, О радиусах устойчивости, квазиустойчивости и стабильности векторной траекторной задачи лексикографической оптимизации, Дискрет. матем., 1998, том 10, выпуск 1, 20-27

DOI: https://doi.org/10.4213/dm409

Использование Общероссийского математического портала Math-Net.Ru подразумевает, что вы прочитали и согласны с пользовательским соглашением http://www.mathnet.ru/rus/agreement

Параметры загрузки:

IP : 3.89 .197 .203

26 апреля 2023 г., 14:41:53 


\title{
О радиусах устойчивости, квазиустойчивости и стабильности векторной траекторной задачи лексикографической оптимизащии
}

\author{
(c) 1998 г. В. А. Емеличев, Р. А. Бердышева
}

\begin{abstract}
Рассматривается многокритериальная задача лексикографической оптимизации на системе подмножеств конечного множества (траекторная задача) с векторной целевой функцией, состоящей из линейных критериев. Исследуются различные типы устоћичвости лексикографического множества при малых изменениях параметров целевых функций. Найдены формулы для вычисления радиусов устоичивости, квазиустоичивости и стабильности в случае чебышевской нормы в пространстве возмущающих параметров.

Работа выполнена при поддержке Фонда фундаментальных исследований Республики Беларусь, проекты Ф 23-70 и Ф 95-70, Международной Соросовской программы образования в области точных наук и фонда DAAD (для первого автора).
\end{abstract}

\section{1. Введение}

Исследованию устойчивости как однокритериальных, так и многокритериальных задач дискретной оптимизации посвящено значительное число работ (см., например, [1-9]). При этом под устойчивостью задачи обычно понимается свойство нерасширяемости либо множества всех оптимальных решений (в однокритериальном случае), либо множества Парето (в многокритериальном случае) при переходе от исходной задачи к возмущенной. В работах $[6,8,9]$ исследуются и другие типы устойчивости многокритериальной дискретной задачи: квазиустойчивость, при которой допускается возможность появления новых паретовских оптимумов при сохранении старых, и стабильность, которая понимается как неизменность всего множества Парето при малых возмущениях параметров задачи.

Широко известные лексикографические задачи оптимизации (см., например, [10-13]) возникают в том случае, когда частные критерии упорядочены (перенумерованы) по важности. В данной работе исследуется устойчивость траекторных задач лексикографической оптимизации. Получены аналоги упомянутых выше результатов для лексикографического множества, являющегося подмножеством множества Парето. 


\section{2. Траекторные задачи}

В схему однокритериальных задач укладываются, как известно, все задачи так называемой комбинаторной оптимизации $[1,2,5]$.

Следуя [6-9], введем понятие $n$-критериальной траекторной задачи.

Пусть $E$ - конечное множество элементов, $T$ - совокупность непустых подмножеств этого множества, называемых траекториями. В дальнейшем будем считать, что число траекторий в $T$ больше единицы. На множестве $E$ зададим векторную весовую функцию

$$
a(e)=\left(a_{1}(e), a_{2}(e), \ldots, a_{n}(e)\right)
$$

где

$$
a_{i}(e) \in \mathbf{R}, \quad i \in N_{n}=\{1,2, \ldots, n\}, \quad e \in E,
$$

а на множестве траекторий $T=\{t\}$ векторный критерий

$$
f(t)=\left(f_{1}(t), f_{2}(t), \ldots, f_{n}(t)\right),
$$

частными критериями которого являются линейные критерии, т.е. критерии вида MINSUM

$$
f_{i}(t)=\sum_{e \in t} a_{i}(e) \rightarrow \min _{T} \quad \forall i \in N_{n}
$$

Если занумеровать все элементы множества $E=\left\{e_{1}, \ldots, e_{m}\right\}$, то функцию $a(e)$ можно представить в виде матрицы $A=\left\{a_{i j}\right\}_{n \times m}$, где $a_{i j}=a_{i}\left(e_{j}\right)$. Изменяя матрицу $A \in \mathbf{R}^{n m}$, будем получать различные векторные весовые функции. Поэтому, если предположить, что множества $E$ и $T$ зафиксированы, то матрица $A$ может служить для индексации векторного критерия $f(t)$, который будем обозначать через $f(t, A)$, а частные критерии через $f_{i}(t, A)$.

В дальнейшем будем использовать обозначение

$$
\tau_{i}\left(t, t^{\prime}, A\right)=f_{i}(t, A)-f_{i}\left(t^{\prime}, A\right) .
$$

Говоря о многокритериальной задаче, обычно имеют в виду задачу поиска одного, нескольких (определенных) или всех элементов паретовского множества.

В данной работе будет исследоваться $n$-критериальная траекторная задача поиска лексикографического множества, которое является подмножеством паретовского множества и определяется следующим образом (см. [10-13]).

Пусть $S_{n}=\{s\}-$ множество всех $n$ ! перестановок чисел $1,2, \ldots, n$. Траекторию $t \in T$ назовем лексикографически оптимальной траекторией (ЛОТ) или лексикографическим оптимумом, если существует такая перестановка $s=\left(s_{1}, s_{2}, \ldots, s_{n}\right) \in$ $S_{n}$, что для любой траектории $t^{\prime} \in T$ выполняется одно из двух условий:

(1) $\tau_{i}\left(t, t^{\prime}, A\right)=0 \forall i \in N_{n}$;

(2) $\exists k \in N_{n} \forall i \in N_{k-1}\left(t_{s_{k}}\left(t, t^{\prime}, A\right)<0 \& \tau_{s_{i}}\left(t, t^{\prime}, A\right)=0\right)$.

В случае, когда $k=1$, последние равенства отсутствуют.

Легко видеть, что всякая ЛОТ является паретовским оптимумом.

Множество всех ЛОТ, определенных для всех $n$ ! перестановок, будем называть лексикографическим множеством и обозначать через $T_{\text {lex }}(A)$, а задачу поиска этого множества через $Z^{n}(A)$. 
Ясно, что обычная траекторная задача дискретной оптимизации (см., например, $[2,5])$ является частным случаем задачи $Z^{n}(A)$ (при $n=1$ ).

Следующие свойства ЛОТ очевидны.

Свойство 1. Траектория $t$ принадлежст $T_{\text {leх }}(A)$, если существует такой индекс $i \in N_{n}$, ито

$$
\tau_{i}\left(t, t^{\prime}, A\right)<0 \quad \forall t^{\prime} \in T, \quad t^{\prime} \neq t
$$

Свойство 2. Траектория $t$ не принадлежит $T_{\text {lех }}(A)$, если для каждого индекса $i \in N_{n}$ существует такая траехтория $t^{\prime} \neq t$, ито $\tau_{i}\left(t, t^{\prime}, A\right)>0$.

\section{3. Радиус устойчивости}

В пространстве $\mathbf{R}^{n m}$ матриц $B=\left\{b_{i j}\right\}_{n \times m}$ зададим Чебышевскую норму

$$
\|B\|=\max \left\{\left|b_{i j}\right|:(i, j) \in N_{n} \times N_{m}\right\} .
$$

Для произвольного числа $\varepsilon>0$ определим множество возмущающих матриц

$$
\mathfrak{R}(\varepsilon)=\left\{B \in \mathbf{R}^{n m}:\|B\|<\varepsilon\right\} .
$$

Пусть $A, B \in \mathbf{R}^{n m}$. Задачу $Z^{n}(A+B)$, полученную из исходной задачи $Z^{n}(A)$ при сложении матриц $A$ и $B$, будем называть возмушенной, а матрицу $B$ возмущающей.

По аналогии с $[7,8]$ радиусом устойчивости задачи $Z^{n}(A)$ назовем число

$$
\rho_{1}^{n}(A)= \begin{cases}\sup Q_{1}(A), & \text { если } Q_{1}(A) \neq \varnothing, \\ 0, & \text { если } Q_{1}(A)=\varnothing,\end{cases}
$$

где

$$
Q_{1}(A)=\left\{\varepsilon>0: T_{\text {lex }}(A+B) \subseteq T_{\text {lex }}(A) \forall B \in \mathfrak{R}(\varepsilon)\right\} .
$$

Таким образом, радиус устойчивости задачи $Z^{n}(A)$ определяет предел возмущений элементов матрицы $A$, при которых не возникает новых лексикографических оптимумов.

Очевидно, что в случае, когда $T=T_{\text {lex }}(A)$, радиус устойчивости $\rho_{1}^{n}(A)$ равен бесконечности. Поэтому этот случай будем исключать из рассмотрения, а задачу $Z^{n}(A)$, для которой $\bar{T}_{\mathrm{lex}}(A)=T \backslash T_{\mathrm{lex}}(A) \neq \varnothing$, будем называть нетривиальной.

Из определения радиуса устойчивости с очевидностью вытекают следующие свойства.

Свойство 3. Пусть $\varphi>0$. Тогда $\rho_{1}^{n}(A) \geqslant \varphi$, если

$$
\bar{T}_{\text {lex }}(A) \subseteq \bar{T}_{\text {lex }}(A+B) \quad \forall B \in \Re(\varphi) .
$$

Свойство 4. Пусть задача $Z^{n}(A)$ нетривиалъна, $\varphi \geqslant 0$. Тогда $\rho_{1}^{n}(A) \leqslant \varphi$, если для любого иисла $\varepsilon>\varphi$ существует возмущающая матрица $B \in \mathfrak{R}(\varepsilon)$ и траектория $t \in \bar{T}_{\mathrm{lex}}(A)$ maxue, umo $t \in T_{\mathrm{lex}}(A+B)$. 
Введем обозначения

$$
\begin{aligned}
& E\left(t, t^{\prime}\right)=t \backslash t^{\prime} \cup t^{\prime} \backslash t, \\
& \Delta\left(t, t^{\prime}\right)=\left|E\left(t, t^{\prime}\right)\right| .
\end{aligned}
$$

Очевидно, что $\Delta\left(t, t^{\prime}\right)>0$, если $t \neq t^{\prime}$.

Теорема 1. Радиус устойчивости нетривиалъной задачи $Z^{n}(A), n \geqslant 1$, выражается формулой

$$
\rho_{1}^{n}(A)=\min _{t \in \bar{T}_{\text {lex }}(A)} \min _{i \in N_{n}} \max _{t^{\prime} \neq t} \frac{\tau_{i}\left(t, t^{\prime}, A\right)}{\Delta\left(t, t^{\prime}\right)} .
$$

Доказательство. Сначала докажем неравенство $\rho_{1}^{n}(A) \geqslant \varphi^{n}(A)$, где через $\varphi^{n}(A)$ обозначена правая часть (1). Это неравенство очевидно, если $\varphi^{n}(A)=0$.

Пусть $\varphi^{n}(A)>0$. Тогда в силу определения числа $\varphi^{n}(A)$ для любой траектории $t \in \bar{T}_{\text {lex }}(A)$ и любого индекса $i \in N_{n}$ существует траектория $t^{\prime} \in T, t^{\prime} \neq t$, такая, что

$$
0<\varphi^{n}(A) \leqslant \frac{\tau_{i}\left(t, t^{\prime}, A\right)}{\Delta\left(t, t^{\prime}\right)} .
$$

Поэтому для всякой матрицы $B \in \mathfrak{B}\left(\varphi^{n}(A)\right)$ в силу неравенства $\|B\|<\varphi^{n}(A)$

$$
\tau_{i}\left(t, t^{\prime}, A+B\right) \geqslant \tau_{i}\left(t, t^{\prime}, A\right)-\sum_{j \in N_{m}: e_{j} \in E\left(t, t^{\prime}\right)}\left|b_{i j}\right| \geqslant \tau_{i}\left(t, t^{\prime}, A\right)-\|B\| \Delta\left(t, t^{\prime}\right)>0 .
$$

Отсюда согласно свойству 2

$$
t \in \bar{T}_{\text {lex }}(A+B) \quad \forall B \in \mathfrak{R}\left(\varphi^{n}(A)\right) .
$$

Следовательно,

$$
\bar{T}_{\text {lex }}(A) \subseteq \bar{T}_{\text {lex }}(A+B) \quad \forall B \in \mathfrak{R}\left(\varphi^{n}(A)\right),
$$

т.е. на основании свойства 3 получаем неравенство $\rho_{1}^{n}(A) \geqslant \varphi^{n}(A)$.

Теперь докажем, что $\rho_{1}^{n}(A) \leqslant \varphi^{n}(A)$. Пусть $\varepsilon>\varphi^{n}(A)$. Тогда, в силу определения числа $\varphi^{n}(A)$, найдутся траектория $t \in \bar{T}_{\text {lex }}(A)$ и индекс $k \in N_{n}$ такие, что

$$
\gamma=\max \left\{\tau_{k}\left(t, t^{\prime}, A\right) / \Delta\left(t, t^{\prime}\right): t^{\prime} \in T, t^{\prime} \neq t\right\}<\varepsilon .
$$

Если в качестве возмущающей матрицы $B \in \mathfrak{R}(\varepsilon)$ возьмем матрицу с элементами

$$
b_{i j}= \begin{cases}-b, & \text { если } e_{j} \in t, i=k, \\ b, & \text { если } e_{j} \notin t, i=k, \\ 0, & \text { если } j \in N_{m}, i \neq k,\end{cases}
$$

где $\gamma<b<\varepsilon$, то с учетом (2) находим, что

$$
\tau_{k}\left(t, t^{\prime}, A+B\right)=\tau_{k}\left(t, t^{\prime}, A\right)-b \Delta\left(t, t^{\prime}\right)<\tau_{k}\left(t, t^{\prime}, A\right)-\gamma \Delta\left(t, t^{\prime}\right) \leqslant 0 \quad \forall t^{\prime} \neq t .
$$

Поэтому согласно свойству $1 t-$ ЛОТ возмущенной задачи $Z^{n}(A+B)$, т.е. на основании свойства 4 получаем, что $\rho_{1}^{n}(A) \leqslant \varphi^{n}(A)$.

Собирая все доказанное, убеждаемся в справедливости теоремы 1. 
Замечание 1. Формула (1) при $n=1$ превращается в известную формулу радиуса устойчивости однокритериальной траекторной задачи с линейными критериями $[1,2]$.

Замечание 2. Формула (2) свидетельствует о том, что бесконечный радиус устойчивос'ги имеет лишь тривиальная задача $Z^{n}(A)$.

Задачу $Z^{n}(A)$ назовем устойчивой, если $Q_{1}(A) \neq \varnothing$, т.е. если существует такое число $\varepsilon>0$, что выполняются включения

$$
T_{\text {lex }}(A+B) \subseteq T_{\text {lex }}(A) \quad \forall B \in \mathfrak{R}(\varepsilon)
$$

Из теоремы 1 вытекает следующее утверждение.

Следствие 1. Нетривиальная задача $Z^{n}(A)$ поиска лексикографического множества $T_{\text {lex }}(A)$ устойчива тогда и толъко тогда, когда

$$
\forall i \in N_{n} \quad \forall t^{\prime} \in \bar{T}_{\text {lex }}(A) \quad \exists t \neq t^{\prime} \quad\left(\tau_{i}\left(t, t^{\prime}, A\right)<0\right) .
$$

Замечание 3. В однокритериальном случае $(n=1)$ аналогом лексикографического множества является множество всех оптимальных траекторий. Поэтому, учитывая следствие 1 , легко видеть, что всякая однокритериальная задача $Z^{1}(A)$ является устойчивой.

\section{4. Радиус квазиустойчивости}

Радиусом квазиустойчивости задачи $Z^{n}(A)$ назовем число

$$
\rho_{2}^{n}(A)= \begin{cases}\sup Q_{2}(A), & \text { если } Q_{2}(A) \neq \varnothing, \\ 0, & \text { если } Q_{2}(A)=\varnothing\end{cases}
$$

где

$$
Q_{2}(A)=\left\{\varepsilon>0: T_{\text {lex }}(A) \subseteq T_{\text {lex }}(A+B) \quad \forall B \in \mathfrak{R}(\varepsilon)\right\} .
$$

Иными словами, такой радиус определяет предел возмуцений элементов матрицы $A$, при которых допускается возможность появления новых ЛОТ при сохранении всех старых (ср. с [9]).

Непосредственно из этого определения вытекают следующие свойства, аналогичные свойствам 3 и 4.

Свойство 5. Пусть $\varphi>0$. Тогда $\rho_{2}^{n}(A) \geqslant \varphi$, если

$$
T_{\text {lex }}(A) \subseteq T_{\text {lex }}(A+B) \quad \forall B \in \mathfrak{R}(\varepsilon) .
$$

Свойство 6. Пусть $\varphi \geqslant 0$. Тогда $\rho_{2}^{n}(A) \leqslant \varphi$, если для любого числа $\varepsilon>\varphi$ существуют траектории $t \in T_{\mathrm{lex}}(A)$ и матрича $B \in \mathfrak{R}(\varepsilon)$ такие, что $t \in \bar{T}_{\mathrm{lex}}(A+B)$. 
Теорема 2. Радиус квазиустойчивости задачи $Z^{n}(A), n \geqslant 1$, выражается формулой

$$
\rho_{2}^{n}(A)=\min _{t \in T_{\text {lex }}(A)} \min _{i \in N_{n}} \max _{t^{\prime} \neq t} \frac{\tau_{i}\left(t, t^{\prime}, A\right)}{\Delta\left(t, t^{\prime}\right)}
$$

Доказательство. Сначала докажем неравенство $\rho_{2}^{n}(A) \geqslant \psi^{n}(A)$, где через $\psi^{n}(A)$ обозначена правая часть (4). Это неравенство очевидно, если $\psi^{n}(A)=0$.

Пусть $\psi^{n}(A)>0$. Тогда $\mathfrak{R}\left(\psi^{n}(A)\right) \neq \varnothing$ и в силу определения числа $\psi^{n}(A)$ для любой ЛОТ $t^{\prime}$ задачи $Z^{n}(A)$ существует индекс $k \in N_{n}$ такой, что

$$
0<\psi^{n}(A) \leqslant \frac{\tau_{k}\left(t, t^{\prime}, A\right)}{\Delta\left(t, t^{\prime}\right)} \quad \forall t \neq t^{\prime} .
$$

Поэтому для всякой возмущающей матрицы $B \in \mathfrak{B}\left(\psi^{n}(A)\right)$

$$
\tau_{k}\left(t, t^{\prime}, A+B\right) \geqslant \tau_{k}\left(t, t^{\prime}, A\right)-\sum_{j \in N_{m}: e_{j} \in E\left(t, t^{\prime}\right)}\left|b_{k j}\right| \geqslant \tau_{k}\left(t, t^{\prime}, A\right)-\|B\| \Delta\left(t, t^{\prime}\right)>0 \quad \forall t \neq t^{\prime} .
$$

Отсюда на основании свойства 1 находим, что

$$
t^{\prime} \in T_{\mathrm{lex}}(A+B) \quad \forall B \in \mathfrak{R}\left(\psi^{n}(A)\right) .
$$

Следовательно, $T_{\text {lex }}(A) \subseteq T_{\text {lex }}(A+B)$, т.е. согласно свойству 5 получаем неравенство

$$
\rho_{2}^{n}(A) \geqslant \psi^{n}(A) \text {. }
$$

Теперь докажем, что $\rho_{2}^{n}(A) \leqslant \psi^{n}(A)$. Пусть $\varepsilon>\psi^{n}(A)$. Тогда, в силу определения числа $\psi^{n}(A)$, найдутся ЛОТ $t^{\prime}$ задачи $Z^{n}(A)$ и траектория $t \neq t^{\prime}$ такие, что

$$
\alpha=\max \left\{\tau_{i}\left(t, t^{\prime}, A\right) / \Delta\left(t, t^{\prime}\right): i \in N_{n}\right\}<\varepsilon .
$$

Если в качестве возмущающей матрицы $B \in \mathfrak{R}(\varepsilon)$ возьмем матрицу с элементами

$$
b_{i j}= \begin{cases}-b, & \text { если } e_{j} \notin t^{\prime}, i \in N_{n}, \\ b, & \text { если } e_{j} \in t^{\prime}, i \in N_{n},\end{cases}
$$

где $\alpha<b<\varepsilon$, то с учетом (5) справедливы неравенства

$$
\tau_{i}\left(t, t^{\prime}, A+B\right)=\tau_{i}\left(t, t^{\prime}, A\right)-b \Delta\left(t, t^{\prime}\right)<\tau_{i}\left(t, t^{\prime}, A\right)-\alpha \Delta\left(t, t^{\prime}\right) \leqslant 0 \quad \forall i \in N_{n} .
$$

Поэтому согласно свойству 2 траектория $t^{\prime} \in \bar{T}_{\text {lex }}(A+B)$, т.е. на основании свойства 6 получаем, что $\rho_{2}^{n}(A) \leqslant \psi^{n}(A)$.

Собирая все доказанное, убеждаемся в справедливости теоремы 2.

Замечание 4. Нетрудно привести примеры матриц $A_{1}, A_{2}$ и $A_{3}$, для которых справедливы соотношения

$$
\rho_{1}^{n}\left(A_{1}\right)<\rho_{2}^{n}\left(A_{2}\right), \quad \rho_{1}^{n}\left(A_{2}\right)=\rho_{2}^{n}\left(A_{2}\right), \quad \rho_{1}^{n}\left(A_{3}\right)>\rho_{2}^{n}\left(A_{3}\right)
$$


Задачу $Z^{n}(A)$ назовем квазиустойчивой, если $Q_{2}(A) \neq \varnothing$, т.е. если существует число $\varepsilon>0$. такое, что выполняются включения

$$
T_{\text {lex }}(A) \subseteq T_{\text {lex }}(A+B) \quad \forall B \in \mathfrak{R}(\varepsilon)
$$

Тогда из теоремы 2 вытекает следующее утверждение.

Следствие 2. Задача $Z^{n}(A)$ квазиустойчива тогда и толъко тогда, когда

$$
\forall t \in T_{\text {lex }}(A) \quad \exists i \in N_{n} \quad \forall t^{\prime} \neq t \quad\left(\tau_{i}\left(t, t^{\prime}, A\right)<0\right) .
$$

\section{5. Стабильность}

Задачу $Z^{n}(A)$ назовем стабильной, если существует такое число $\varepsilon>0$, что выполняются равенства

$$
T_{\text {lex }}(A+B)=T_{\text {lex }}(A) \quad \forall B \in \mathfrak{R}(\varepsilon) .
$$

Очевидно, что задача $Z^{n}(A)$ стабильна тогда и только тогда, когда она одновременно устойчива и квазиустойчива. Поэтому ввиду следствий 1 и 2 справедливо следующее утверждение.

Следствие 3. Задача $Z^{n}(A)$ стабилъна тогда и толъко тогда, когда справедливъ формуль (3) и (6).

Кроме того, нетрудно убедиться, что задача $Z^{n}(A)$ стабильна, если

$$
\forall i \in N_{n} \quad \exists t \in T_{\text {lex }}(A) \quad \forall t^{\prime} \neq t \quad\left(\tau_{i}\left(t, t^{\prime}, A\right)<0\right) .
$$

Для радиуса стабильности задачи $Z^{n}(A)$, который естественно определить как число

$$
\rho_{\mathbf{3}}^{n}(A)= \begin{cases}\sup Q_{3}(A), & \text { если } Q_{3}(A) \neq \varnothing \\ 0, & \text { если } Q_{3}(A)=\varnothing\end{cases}
$$

где

$$
Q_{\mathbf{3}}(A)=\left\{\varepsilon>0: T_{\text {lex }}(A)=T_{\text {lex }}(A+B) \quad \forall B \in \mathbf{R}(\varepsilon)\right\}
$$

очевидно соотношение

$$
\rho_{3}^{n}(A)=\min \left\{\rho_{1}^{n}(A), \rho_{2}^{n}(A)\right\}
$$

Замечание 5. С учетом эквивалентности любых двух норм в конечномерном линейном пространстве (см. [14]) следствия 1,2 и 3 справедливы не только для Чебышевской, но и для других норм в пространстве возмущающих матриц. 


\section{Список литературы}

1. Леонтьев В. К. Устойчивость в линейных дискретных задачах. Пробл. киберн. (1979) 35, 169-184.

2. Леонтьев В. К., Гордеев Э. Н. Качественнпе исследование траекторных задач. Киберremuxa (1986) №5, 82-89.

3. Гордеев Э. Н. Полиномиальные алгоритмы вычисления радиуса устойчивости для двух классов задач выбора. Докл. АН CCCP (1987) 297, №5, 1040-1043.

4. Гордеев Э. Н. Обб устойчивости задач на узкие места. Журнал вычисл. матем. и матем. физики (1993) 33, №9, 1391-1402.

5. Гордеев Э. Н., Леонтьев В. К. Общий подход к исследованию устойчивости решений в задачах дискретноң оптимизации. Журнал вычисл. матем. и матем. физики (1996) 36, №1, 66-72.

6. Казерацкая А. Н., Лебедева Т. Т., Сергиенко Т. И. Исследование устойчивости задач дискретной оптимизации. Кибернетика и системный аяализ (1993) №4, 78-91.

7. Бакурова А. В., Емеличев В. А., Перепелица В. А. Об устойчивости многокритериальных задач на системах подмножеств. Докл. АН Беларуси (1995) 39, №2, 33-35.

8. Емеличев В. А., Кравцов М. К. Об устойчивости в траекторных задачах векторной оптимизации. Кибернетиха и системнъй анализ (1995) №4, 137-143.

9. Емеличев В. А., Кравцов М. К., Подкопаев Д. П. О радиусе квазиустойчивости многокритериальной траекторной задачи. Докл. АН Беларуси (1996) 40, №1, 9-12.

10. Подиновский В. В., Гаврилов В. М. Оптимизачия по последователъно применяемъм критериям. Советское радио, Москва, 1975.

11. Подиновский В. В., Ногин В. Д. Парето-оптималънъе решения многохритериалъных задач. Наука, Москва, 1982.

12. Сергиенко И. В. Математические модели и методы решения задач дискретной оптимизачии. Наукова думка, Киев, 1985.

13. Перепелица В. А., Сергиенко И. В. Исследование одного класса целочисленных многокритериальных задач. Журнал вычисл. матем. и матем. физики (1988) 28, №3, 400-419.

14. Колмогоров А. Н., Фомин С. В. Элементы теории фунхций и фунхционалъного аяализа. Наука, Москва, 1972. 